Anestesiología

Abril-Junio 2021

Vol. 44. No. 2. pp 98-104

doi: $10.35366 / 99012$

\section{Eficacia terapéutica de la buprenorfina para tratamiento de dolor agudo postoperatorio en cirugía torácica por toracoscopía}

\author{
Therapeutic efficacy of buprenorphine for treatment of acute \\ postoperative pain in thoracic surgery by thoracoscopy \\ Dr. Juan José Posso-Sierra,* Dr. Orlando Carrillo-Torres, ${ }^{\ddagger}$ \\ Dr. José Damián Carrillo-Ruiz, ${ }^{\ddagger}$ Dra. Verónica de La Vega-Montiel, ${ }^{\ddagger}$ \\ Dra. Claudia I Gutiérrez-Román*
}

\begin{abstract}
Citar como: Posso-Sierra JJ, Carrillo-Torres O, Carrillo-Ruiz JD, de La Vega-Montiel V, Gutiérrez-Román Cl. Eficacia terapéutica de la buprenorfina para tratamiento de dolor agudo postoperatorio en cirugía torácica por toracoscopía. Rev Mex Anestesiol. 2021; 44 (2): 98-104. https://dx.doi.org/10.35366/99012
\end{abstract}

\begin{abstract}
RESUMEN. Introducción: El dolor postoperatorio en toracoscopía tiene una incidencia del 80\%. Estos procedimientos se realizan bajo anestesia general o sedoanalgesia. La buprenorfina en dosis de $4 \mu \mathrm{g} / \mathrm{kg}$ o más es un fármaco pilar para el tratamiento de dolor postoperatorio; sin embargo, dosis menores pueden ser suficientes. El objetivo de este estudio fue demostrar que dosis de 1-3 $\mu \mathrm{g} / \mathrm{kg}$ de buprenorfina aporta una adecuada analgesia con menores efectos adversos durante el postoperatorio en pacientes sometidos a cirugía toracoscópica. Material y métodos: Se realizó el estudio prospectivo, transversal y doble ciego en 48 pacientes sometidos a toracoscopía, divididos en 3 grupos: A $(1 \mu \mathrm{g} / \mathrm{kg}), \mathrm{B}(2 \mu \mathrm{g} / \mathrm{kg})$ y C $(3 \mu \mathrm{g} / \mathrm{kg})$ tratados con buprenorfina, se evaluaron efectos adversos, intensidad de dolor por escala verbal numérica (EVN) y sedación con escala de agitación-sedación de Richmond (RASS) de los 0 a los 120 minutos postanestesia. Resultados: Las dosis de 1 a $3 \mu \mathrm{g} / \mathrm{kg}$ de buprenorfina no ocasionaron efectos adversos. El mantenimiento de analgesia es mayor con $2 \mu \mathrm{g} / \mathrm{kg}(p=0.019)$ y $3 \mu \mathrm{g} / \mathrm{kg}(p=0.045)$. Las dosis de 1 y $2 \mu \mathrm{g} / \mathrm{kg}$ presentan un nivel de sedación menor. Conclusiones: La analgesia multimodal disminuyó un $200 \%$ el dolor postquirúrgico inmediato. Las dosis de 2-3 $\mu \mathrm{g} / \mathrm{kg}$ de buprenorfina son efectivas para el control del dolor con menor incidencia de efectos adversos y un nivel de sedación superficial durante el postoperatorio.
\end{abstract}

ABSTRACT. Introduction: Posterior pain in thoracoscopy has an $80 \%$ incidence. These procedures are done under general anesthesia or sedoanalgesia. The buprenorphin in dosage of $4 \mu \mathrm{g} / \mathrm{kg}$ it's the foundation for post-operatory pain treatment, nevertheless, smaller dosage can be sufficient. The objective of this study is to demonstrate that a dosage of buprenorphin from 1-3 $\mu \mathrm{g} / \mathrm{kg}$ provides suitable analgesia with less side effects during post-operatory in patients who underwent thoracoscopic surgery. Material and methods: We conducted a double-blind, prospective, transversal study, in 48 patients which underwent a thoracoscopy divided in 3 groups: A $(1 \mu \mathrm{g} / \mathrm{kg}), B(2 \mu \mathrm{g} / \mathrm{kg})$, and C (3 $\mu \mathrm{g} / \mathrm{kg})$ treated with buprenorphin. We studied side effects, pain intensity (NVE), and sedative effect (RASS) from 0 to 120 minutes postanesthesia. Results: Dosage from 1 to $3 \mu \mathrm{g} / \mathrm{kg}$ did not generate side effects. The analgesia effect is higher with $2 \mu \mathrm{g} / \mathrm{kg}(p=0.019)$ and $3 \mu \mathrm{g} / \mathrm{kg}(p=0.045)$. Dosage of 1 and $2 \mu \mathrm{g} / \mathrm{kg}$ have lower sedative effect. Conclusion: The multimodal analgesia was diminished by $200 \%$ inmidiate postsurgical pain. Dosage from 2-3 $\mu \mathrm{g} / \mathrm{kg}$ of buprenorphine are effective for pain control with less side effects and a superficial sedative effect during post-operatory.

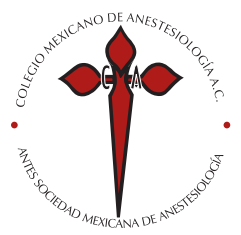

Palabras clave: Dolor postoperatorio, toracoscopía, buprenorfina, analgesia multimodal.

Keywords:

Postoperatory pain, thoracoscopy, buprenorphin, multimodal analgesia.

* Médico residente de

Anestesiología.

‡ Médico adscrito de Anestesiología.

Hospital General de México «Dr. Eduardo Liceaga».

Correspondencia:

Dra. Claudia I Gutiérrez-Román Calle Xochicalco Núm. 30, Dpto. 2a Col. Narvarte Poniente, 03020 Alcaldía Benito Juárez, CDMX Tel: 55 4193-3712

E-mail: claudiagtzr23@gmail.com

Recibido: 13-01-2020

Aceptado:17-08-2020

Abreviaturas:

BIS = Índice Biespectral. $\mathrm{ECG}=$ Electrocardiograma . EVN = Escala verbal numérica. PANI $=$ Presión arterial no invasiva. RASS = Richmond AgitationSedation Scale. UCPA = Unidad de Cuidados Postanestésicos. UTI $=$ Unidad de Cuidados Intensivos.

\section{INTRODUCCIÓN}

$\mathrm{S}^{\mathrm{e}}$ egún la Sociedad Americana de Anestesiología (ASA, por sus siglas en inglés), el dolor agudo relacionado con una cirugía es aquel que se presenta en el paciente quirúrgico posterior al procedimiento. Sin embargo, debemos tomar en cuenta que el dolor postquirúrgico se caracteriza por diversas experiencias sensoriales, emocionales y mentales desagradables, asociadas con respuestas autonómicas, endocrinometabólicas, fisiológicas y conductuales ${ }^{(1-3)}$.

En la actualidad, el dolor agudo postoperatorio tiene una alta incidencia correspondiendo hasta al 50\% en cirugía no torácica y de hasta el $80 \%$ cirugía de tórax y 
ortopédica. Los factores predictivos del dolor postoperatorio resultante de diversos procedimientos quirúrgicos son principalmente el dolor preoperatorio, ansiedad, edad, tipo de cirugía (abdominal, ortopédica o torácica) y duración (Figura 1) $)^{(4,5)}$.

La sensibilización central juega un papel importante en el dolor postquirúrgico, el cual es principalmente nociceptivo somático resultado de la agresión quirúrgica. Posterior a la agresión presentada en los receptores nociceptivos durante el evento quirúrgico, se presenta un estado hiperalgésico. Éste se divide en hiperalgesia primaria, resultado de la sensibilización de los nociceptores periféricos; e hiperalgesia secundaria, que se encuentra asociada con la sensibilización de la médula espinal y el sistema nervioso central. En caso de perpetuarse la nocisepción, se presentan alteraciones como alodinia, parestesias, etcétera. Bayman demostró que la toracoscopía provoca hasta un $25 \%$ de dolor crónico ${ }^{(5-8)}$.

Para poder tratar el dolor agudo postoperatorio se debe realizar una evaluación objetiva del mismo, a través de métodos y escalas dentro de las cuales se encuentra la EVN, es el método de medición empleado con más frecuencia en muchos centros de evaluación del dolor, la cual consta de la referencia del paciente tomando a 0 como ausencia de dolor y 10 como máximo dolor ${ }^{(9-11)}$.

La primera línea para el tratamiento de dolor postquirúrgico son los opioides. La buprenorfina es un agonista parcial de opiáceos derivado de la tebaína, sintetizado en 1969 por KW Bentley, tiene una estructura hexacíclica y fue comercializada por primera vez en los Estados Unidos en la década de 1980 por los laboratorios Reckitt \& Colman como analgésico, con el nombre comercial de Buprenex $0.3 \mathrm{mg} / \mathrm{mL}$ inyectable. En comparación con la morfina, es más liposoluble con una potencia 20 a 30 veces superior, convirtiéndose en una molécula atractiva para la aplicación por diferentes vías, intravenosa, sublingual, transdérmica. Las dosis recomendadas para el manejo del dolor postquirúrgico por grupos de edades son: 2-12 años de 2-6 $\mu \mathrm{g} / \mathrm{kg}$ cada seis a ocho horas, $\geq 13$ años de 3 a $6 \mu \mathrm{g} / \mathrm{kg}$ en intervalos de hasta cada seis horas. Los efectos adversos más frecuentes son náuseas y vómitos y se presenta en relación a la dosis. Se ha observado que dosis menores de $4 \mu \mathrm{g} / \mathrm{kg}$ son efectivas y con pocas probabilidades de náusea y vómito ${ }^{(12-17)}$.

La toracoscopía es un procedimiento que se basa en la colocación de un telescopio (toracoscopio) dentro de la cavidad torácica para la visualización del tórax en tiempo real, se puede realizar bajo anestesia local más sedación o anestesia general balanceada. Se ha descrito ampliamente el uso de propofol y midazolam para sedación en estos procedimientos, así como fentanilo y bloque peridural o intercostal para el control analgésico. Sin embargo, Grendelmeier y colaboradores ${ }^{(18)}$ encontraron mayor riesgo al utilizar propofol vs midazolam para sedación consciente, dada una mayor incidencia de hipoxemia (saturación de oxígeno arterial pulsátil < 90\%) y episodios de hipotensión (presión arterial sistólica $<90 \mathrm{mmHg})^{(8,19-22)}$. El uso de buprenorfina aún carece de evidencia científica que describa la dosis eficaz para efectos secundarios comunes como náuseas y/o vómitos en recuperación postquirúrgica o en pabellón. En este estudio se evaluó la eficacia terapéutica de la buprenorfina al comparar tres dosis del medicamento, observando como objetivo secundario el control de la náusea y vómito postoperatorio para cirugía toracoscópica en paciente despierto.

\section{MATERIAL Y MÉTODOS}

Se realizó un estudio prospectivo, transversal, doble ciego a 48 pacientes sometidos a toracoscopía. Para el cálculo de

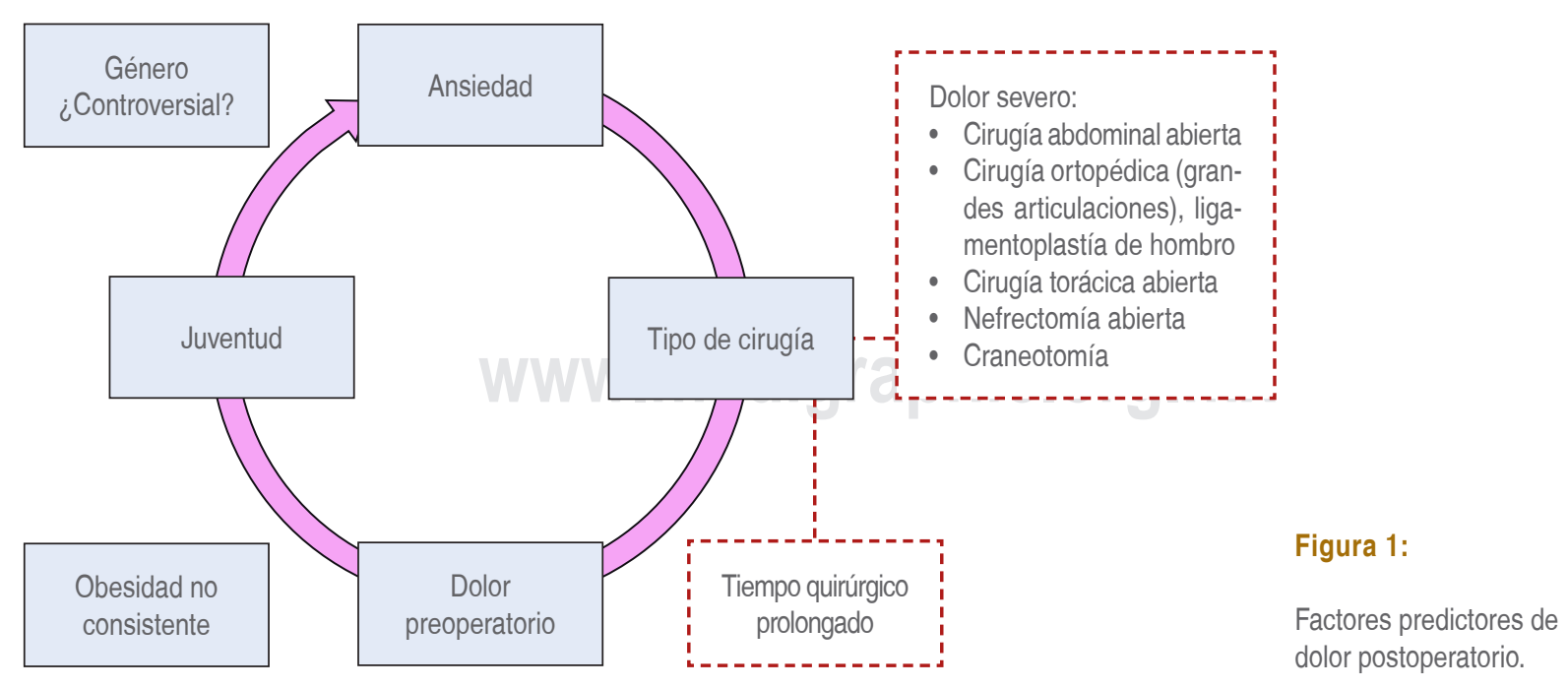




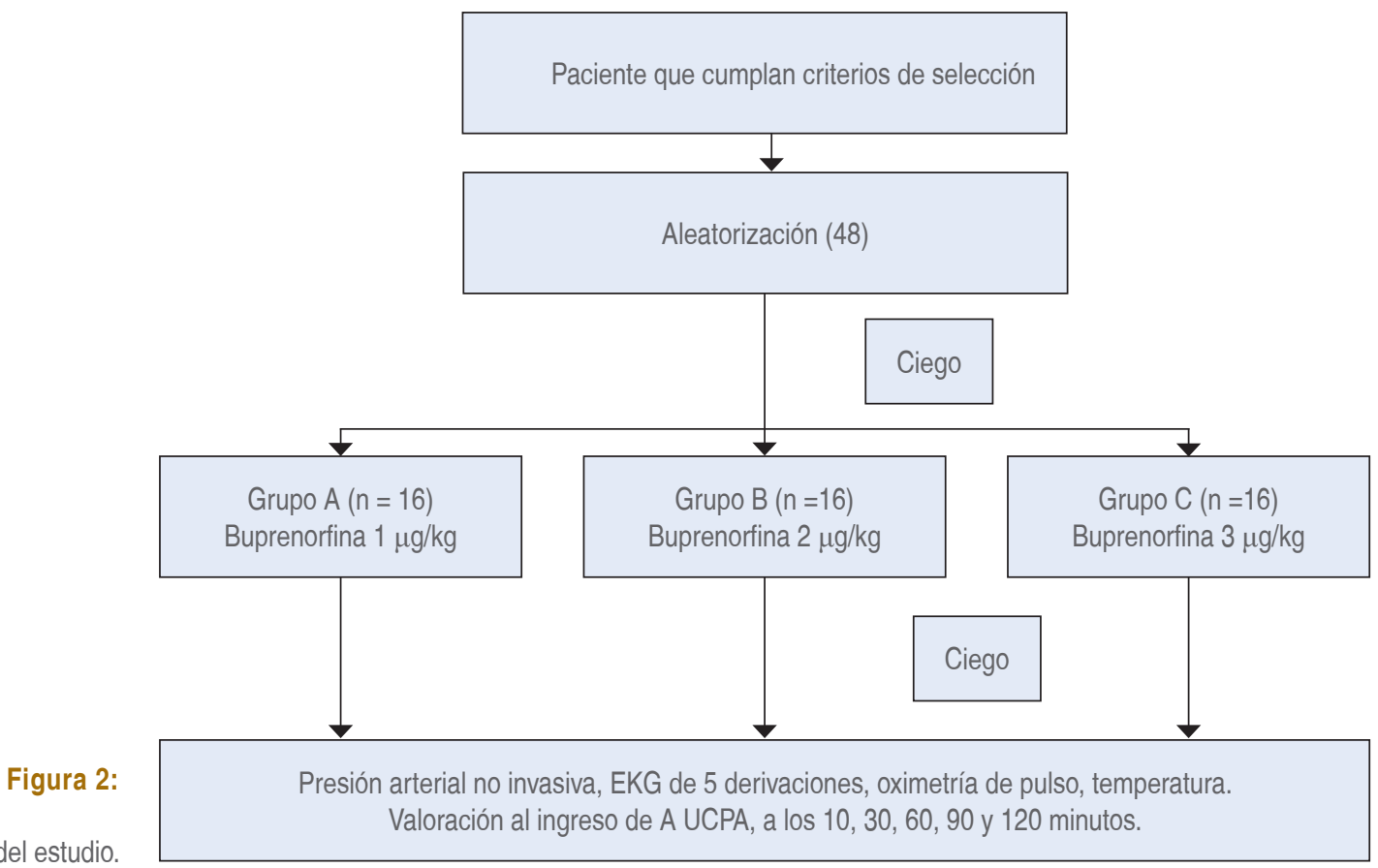

Flujograma del estudio.

Frecuencia de cirugías toracoscópicas

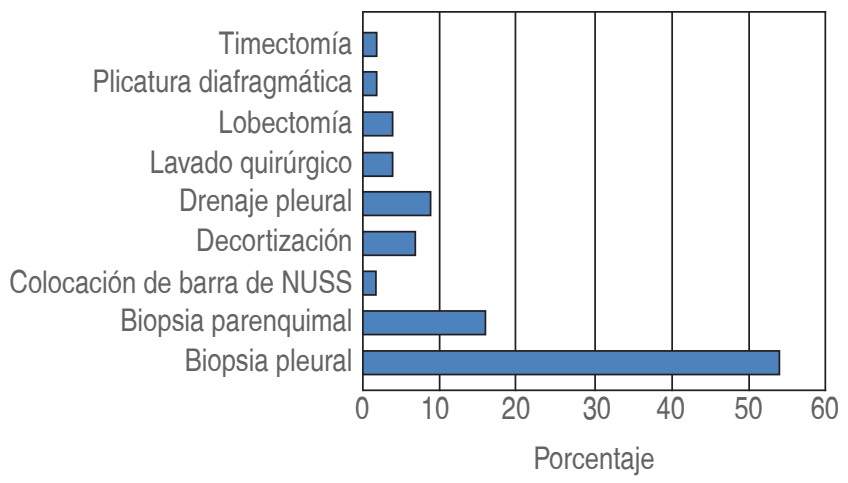

Figura 3: Frecuencia del tipo de cirugía realizada: biopsia pleural 25 pacientes, biopsia de parénquima pulmonar siete pacientes, colocación de barra de Nuss uno, decorticación tres, drenaje pleural cuatro, lavado quirúrgico dos, lobectomía dos, plicatura diafragmática uno, timectomía uno.

muestra se tomó en cuenta a la población ingresada al Servicio de Neumología del Hospital General de México para cirugía de toracoscopía en el año $2016(\mathrm{~N}=62)$. Se estimó un error alfa bilateral de 0.05 con error beta de 0.20 y una pérdida probable de pacientes del $10 \%$ de la muestra con el paquete estadístico $\mathrm{G}$ power.

Este estudio se condujo de acuerdo a las normas de ética sobre investigación en sujetos humanos de la Declaración de Helsinki, el reglamento de la Ley General de Salud en materia de investigación para la salud. El protocolo fue autorizado por el Comité de Ética e Investigación del Hospital General de México «Dr. Eduardo Liceaga» con número de aprobación de DI/17/203/3/050.

Procedimiento: después de firmar consentimientos informados para realizar procedimiento anestésico, los pacientes ingresaron a sala de quirófano, donde se monitorizaron con presión arterial no invasiva (PANI), frecuencia cardíaca, electrocardiograma (EKG) de cinco derivaciones, frecuencia respiratoria, índice biespectral (BIS) y pulsioximetría. En caso de no existir contraindicaciones médicas para el procedimiento, se realizó toracoscopía con paciente despierto. A todos los pacientes se les proporcionaron los siguientes medicamentos durante el transanestésico: bloqueo con ropivacaína al $0.2 \%$ en zona intercostal y en herida quirúrgica; propofol dosis respuesta (para mantener un BIS entre 40 y 60\%); fentanilo IV concentración plasmática 2-4 ng/mL a una perfusión de $0.026-0.052 \mu \mathrm{g} / \mathrm{kg} / \mathrm{min}$; lidocaína a $25 \mu \mathrm{g} /$ $\mathrm{kg} / \mathrm{min} \mathrm{IV}$; metamizol $30 \mathrm{mg} / \mathrm{kg}$ IV; dexametasona $8 \mathrm{mg}$ IV; ondansetrón 8 mgs IV. Treinta minutos previos al término de la cirugía, se aplicó buprenorfina en perfusión intravenosa durante cinco minutos con bomba de jeringa-pistón de la siguiente manera: grupo A $1 \mu \mathrm{g} / \mathrm{kg}$, grupo B $2 \mu \mathrm{g} / \mathrm{kg}$ y grupo C $3 \mu \mathrm{g} / \mathrm{kg}$, después se vigilaron en la Unidad de Cuidados Postanestésicos monitorizados con PANI, EKG de 5 derivaciones, pulsioximetría y temperatura. Se llevó el registro de la analgesia obtenida en el postoperatorio además de las otras variables secundarias a los cero, 10, 30, 60, 90 y 120 minutos. En caso de dolor, se establece el siguiente rescate analgésico: ENV 1-3 no se administran medicamentos; 
Tabla 1: Frecuencia de intensidad de dolor (EVN) al ingreso a Unidad de Cuidados Postanestésicos.

\begin{tabular}{crrrrr} 
Grupo n (\%) & 0 & 1 & 2 & 3 & 4 \\
\hline A & $2(13.30)$ & $9(60.00)$ & $3(20.00)$ & $0(0)$ & $1(6.7)$ \\
B & $4(26.66)$ & $10(66.67)$ & $1(6.67)$ & $0(0)$ & $0(0.0)$ \\
C & $6(37.50)$ & $8(50.00)$ & $2(12.50)$ & $0(0)$ & $0(0.0)$ \\
\hline
\end{tabular}

Observamos la intensidad de dolor en el momento en el que el paciente ingresa a Unidad de Cuidados Postanestésicos tanto en número $(n)$ como en porcentaje (\%), expresado con base a la escala verbal numérica, en cada grupo (grupo $A=1 \mu \mathrm{g} / \mathrm{kg}$, grupo $B=2 \mu \mathrm{g} / \mathrm{kg}$, grupo $\mathrm{C}=3 \mu \mathrm{g} / \mathrm{kg}$ ).

ENV 4-5 se administra buprenorfina a un tercio de la dosis utilizada para analgesia postoperatoria vía IV; con EVN de 6 a 10 se administra buprenorfina IV a la mitad de la dosis utilizada para analgesia postoperatoria, la intensidad del dolor fue evaluada nuevamente a los 10 minutos en todos los casos (Figura 2).

Para mantener el estudio cegado un investigador aplicó la buprenorfina, realizó el procedimiento anestésico y recogió los datos en el período postquirúrgico, un segundo investigador realizó las diluciones y la aleatorización y un tercer investigador realizó el análisis estadístico.

Análisis estadístico: para la evaluación de la variable primaria «intensidad de dolor» (EVN) y sedación (RASS), se utilizó la prueba de Shapiro-Wilk que determinó que las variables fueron no paramétricas, posteriormente se llevó a cabo un análisis de medias para pruebas no paramétricas por medio de la prueba Kruskal-Wallis.

Por medio de la prueba de t de Student para variables dependientes, se compararon medias para conocer la diferencia entre variables cuantitativas continuas durante el tiempo, tanto para el dolor (EVN) como para sedación (RASS). Para las variables cualitativas se usó estadística descriptiva.

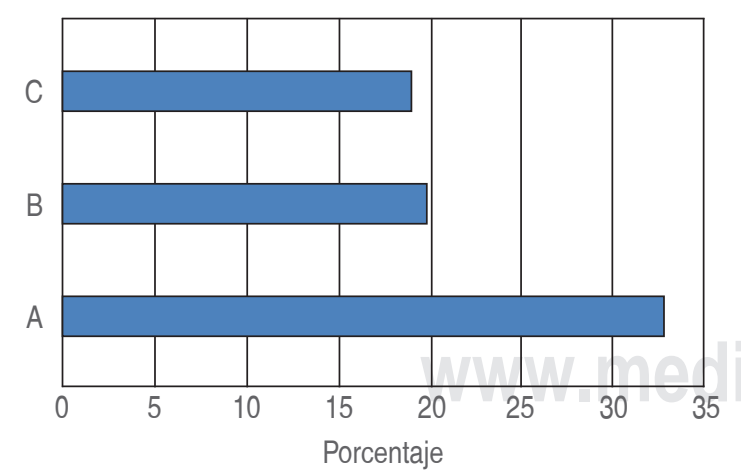

Figura 4: Frecuencia de pacientes con descontrol algológico. Se consideró descontrol algológico un EVN de cuatro o más, observándose mayor descontrol algológico en el grupo $A$. (Grupo $A=1 \mu \mathrm{g} / \mathrm{kg}$, grupo $B=$ $2 \mu \mathrm{g} / \mathrm{kg}$, grupo $\mathrm{C}=3 \mu \mathrm{g} / \mathrm{kg}$ ).

$E V N=$ escala verbal numérica.

\section{RESULTADOS}

De los 48 pacientes, se eliminó uno del grupo A y uno del grupo $\mathrm{B}$, ya que ambos egresaron a la Unidad de Terapia Intensiva (UTI). Los casos estudiados tenían una edad de entre 20 y 79 años con una media de 53 años, el género fue predominantemente masculino (72\%), mientras que el femenino fue de $28 \%$; el procedimiento más frecuente fue la toma de biopsia (Figura 3).

En cuanto a las variables dependientes analizadas, ningún paciente presentó náusea y/o vómito, RASH y/o depresión respiratoria. El control del dolor posoperatorio inmediato no controlado se observó en un paciente del grupo A (Tabla 1); al finalizar las dos horas de cuidados postquirúrgicos, se evidenció una frecuencia de descontrol de dolor agudo (EVN de cuatro o mayor) en el 33\% de los pacientes del grupo A, y de 20 y $18.7 \%$ del grupo $\mathrm{B}$ y $\mathrm{C}$ respectivamente (Figura 4). Sin embargo, no se demostraron diferencias estadísticamente significativas en las medias para la intensidad de dolor en el momento de ingreso a la Unidad de Cuidados Postanestésicos (UCPA). En cuanto a la intensidad de dolor en relación al tiempo de estancia, se demostró la aparición más temprana (a los 10 minutos) estadísticamente significativa en el grupo A, en comparación al grupo B y C (Tabla 2).

Para la evaluación de sedación se utilizó la escala RASS, ningún paciente presentó RASS de -4 (sedación profunda) o -5 (sin respuesta). El grupo que presentó menor frecuencia de sedación moderada (-3) fue el grupo A, sin diferencias estadísticamente significativas entre grupos a su ingreso a UCPA (Figura 5). En cuanto a la duración de la sedación por tiempo, observamos una recuperación lenta estadísticamente significativa en el grupo C comparado con el grupo A y B (Tabla 3).

\section{DISCUSIÓN}

Novedad en el estudio: no existen estudios previos que valoren estas dosis en ningún tipo de escenario, además de ser el primer estudio que compara buprenorfina en el período postquirúrgico con pacientes despiertos bajo un procedimiento toracoscópico, por ello, no se pueden realizar comparaciones 
Tabla 2: Diferencias de medias en intensidad de dolor conforme el paso de tiempo.

\begin{tabular}{|c|c|c|c|c|c|c|}
\hline Grupo & $0^{\prime}$ & $10^{\prime}$ & $30^{\prime}$ & $60^{\prime}$ & $90^{\prime}$ & 120' \\
\hline$A$ & 1 & $0.041^{*}$ & 0.02 & $<0.000$ & $<0.000$ & $<0.000$ \\
\hline B & 1 & 0.164 & $0.019 *$ & 0.006 & 0.003 & $<0.000$ \\
\hline C & 1 & 0.384 & $0.045^{*}$ & 0.012 & 0.004 & 0.001 \\
\hline
\end{tabular}

Evaluación de la intensidad de dolor medido por (EVN) a los $0,10,30,60,90$ y 120 minutos de estancia en UCPA por grupos (grupo A $=1 \mu \mathrm{g} / \mathrm{kg}, \mathrm{grupo} B=2 \mu \mathrm{g} / \mathrm{kg}, \mathrm{grupo} \mathrm{C}=3 \mu \mathrm{g} / \mathrm{kg}$ ), realizado por ANOVA.

EVN = escala verbal numérica, UCPA = Unidad de Cuidados Posanestésicos. ANOVA = Análisis de varianza.

${ }^{*}$ Estadísticamente significativo.

con otros trabajos. Se encontró que utilizar dosis de 1, 2 o 3 $\mu \mathrm{g} / \mathrm{kg}$ cuando se analizan las frecuencias presenta un ligero aumento de pacientes con dolor a la utilización de $1 \mu \mathrm{g} / \mathrm{kg}$, pero no conlleva una mejor analgesia al valorar la significancia estadística.

Eficacia anestésica de la buprenorfina a diferentes dosis con analgesia multimodal: aunque en un inicio se pensaba que la toracoscopía al disminuir la longitud de la herida quirúrgica provocaba muy poco dolor, $\operatorname{Bayman}^{(8)}$ en el estudio realizado en el 2017, donde se realizaron toracotomías y toracoscopías con intubación selectiva e infiltración de los nervios intercostales y anestesia general balanceada a base de opioides, demostró que la toracotomía y la toracoscopía son cirugías con un nivel de dolor semejante en los primeros tres días con una media para la toracoscopía de 6.5 (dolor intenso) en escala de EVA a los 120 minutos posteriores al ingreso a la UCPA. En este estudio se demostró que el utilizar una analgesia multimodal con bloque intercostal e infiltración de la herida con anestésico local, más el uso de fármacos con sinergia analgésica; como son paracetamol, glucocorticoide, metamizol, opioide transanestésico y buprenorfina antes del egreso mantiene una media de intensidad de dolor entre $2.5 \mathrm{y}$ 2.8 , o sea, alrededor de $200 \%$ veces menos que lo encontrado por Bayman. Pergolozzi(17) en el año de 2012 describió las dosis recomendadas del fármaco para control de dolor postquirúrgico, llegando a utilizar hasta $300 \mu \mathrm{g}$ por dosis cada seis horas, en el presente estudio se demostró que con dosis bajas del medicamento ( 1 a $3 \mu \mathrm{g} / \mathrm{kg}$ ) es suficiente para un adecuado control del dolor.

Al analizar la cantidad de dolor conforme el paso del tiempo, se encontró que con dosis de $1 \mu \mathrm{g} / \mathrm{kg}$ hay una diferencia estadísticamente significativa en las medias de dolor al ingreso a UCPA y las presentadas al minuto $10(\mathrm{p}=0.41)$ en comparación con las otras dosis que lo presentan hasta el minuto 30, por lo que el mantenimiento de analgesia a través del tiempo es más constante con $2 \mu \mathrm{g} / \mathrm{kg}(\mathrm{p}=0.019)$ y $3 \mu \mathrm{g} /$ $\mathrm{kg}(\mathrm{p}=0.045)$.

Sedación con diferentes dosis de buprenorfina: en cuanto a sedación, las frecuencias encontradas al ingreso a la UCPA demuestran menor escala de RASS para los pacientes del grupo C, pero al analizarse diferencias de medias no fue estadísticamente significativo. Al analizarse conforme el paso del tiempo, se encontró que una dosis de $3 \mu \mathrm{g} / \mathrm{kg}$ produce una disminución estadísticamente significativa en la sedación desde el minuto $10(\mathrm{p}=0.041)$ a diferencia del grupo de $2 \mu \mathrm{g} / \mathrm{kg}$ que lo presenta al minuto $30(\mathrm{p}=0.041)$ y del grupo de $1 \mu \mathrm{g} /$ kg que lo presenta en el minuto 90 ( $\mathrm{p}=0.028)$; así, con dosis de 1 y $2 \mu \mathrm{g} / \mathrm{kg}$ se mantienen con un nivel de sedación similar a la valorada durante el ingreso y durante los 120 minutos de estancia en la UCPA.

Diferencias en los efectos colaterales: se ha descrito que el uso de dosis moderadamente altas de buprenorfina como analgésico postoperatorio producen efectos que van desde náusea hasta depresión respiratoria, en este trabajo se evidenció que a dosis que van de 1 a $3 \mu \mathrm{g} / \mathrm{kg}$ de buprenorfina, con el esquema de analgesia multimodal, no se presentó en ningún caso náuseas/vómitos y/o depresión respiratoria.

Aplicabilidad de la analgesia para pacientes sometidos a procedimientos toracoscópicos: la buprenorfina, por sus propiedades farmacológicas $^{(23,24)}$, puede utilizarse en pacientes con daño renal, daño hepático e inmunodeprimidos, por

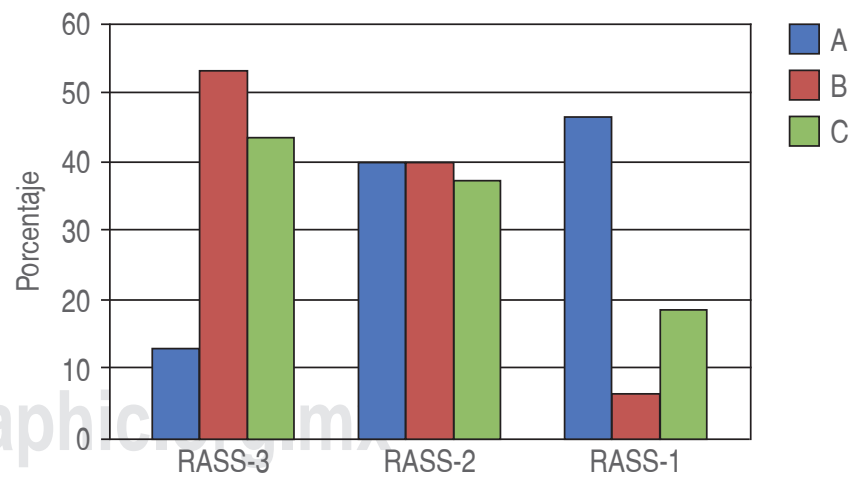

Figura 5: Nivel de sedación (RASS) al ingreso a UCPA. Nivel de Sedación representado en frecuencia, evaluado por escala de RASS al momento de ingreso a UCPA por grupo (Grupo A en color azul = $1 \mu \mathrm{g} / \mathrm{kg}$, Grupo B en color rojo $=2 \mu \mathrm{g} / \mathrm{kg}$ y Grupo C en color verde $=3 \mu \mathrm{g} / \mathrm{kg}$ ).

RASS $=$ Richmond Agitation-Sedation Scale,

UCPA = Unidad de Cuidados Postanestésicos. 
Tabla 3: Diferencia de nivel de sedación RASS entre las medias conforme el tiempo, durante su estancia en Unidad de Cuidados Postanestésicos.

\begin{tabular}{ccccrrr} 
Grupo & $0^{\prime}$ & $10^{\prime}$ & $30^{\prime}$ & $60^{\prime}$ & $90^{\prime}$ & $120^{\prime}$ \\
\hline A & 1 & 0.544 & 0.491 & 0.362 & $0.028^{*}$ & $<0.000$ \\
B & 1 & 0.164 & $0.041^{*}$ & $<0.000$ & $<0.000$ & $<0.000$ \\
C & 1 & $0.041^{*}$ & 0.004 & $<0.000$ & $<0.000$ & $<0.000$ \\
\hline
\end{tabular}

Evaluación de la profundidad de sedación evaluado con la escala de RASS a los $0,10,30,60,90$ y 120 minutos de estancia en UCPA por grupos (grupo A $=1 \mu \mathrm{g} / \mathrm{kg}$, grupo B $=2 \mu \mathrm{g} / \mathrm{kg}$, grupo $\mathrm{C}=3 \mu \mathrm{g} / \mathrm{kg}$ ), realizado por ANOVA.

RASS = Richmond Agitation-Sedation Scale, UCPA = Unidad de Cuidados Posanestésicos, ANOVA = Análisis de varianza

${ }^{*}$ Estadísticamente significativo.

lo que, a diferencia de otros opioides, no constituye peligro con cambios en el funcionamiento fisiológico del paciente en quirófano. La potencia con la que cuenta ( 30 a 40 veces mayor que la morfina) produce una adecuada analgesia mientras los nervios intercostales sean bloqueados y se ministren diversos medicamentos como paracetamol, dexametasona y metamizol durante la toracoscopía, ya que el estímulo principal es en la zona por donde los trócares y la cámara óptica ingresan a la cavidad torácica.

Costo-efectividad de la analgesia con buprenorfina vs diferente tipo de analgesia en pacientes sometidos a toracoscopía: en México, la Secretaría de Salud elaboró un módulo de estimación de costos que se actualiza aproximadamente cada cinco años. En el 2014, los costos por hospitalización en unidades de terapia intensiva, donde la mayor parte de estos pacientes egresa sobre todo si aún no cuenta con un control adecuado del dolor o está intubado y bajo ventilación mecánica, varían desde $\$ 910.00$ en salubridad hasta $\$ 7,800.00$ en un hospital privado tipo A1. Esto sin contar con los gastos inherentes al cuidado de un paciente crítico, con lo que puede aumentar en un $1,000 \%$. Es probable que los pacientes no requieran cuidados intensivos pero sí intermedios si fueron intubados y extubados o presentan analgesia parcial, el monto por cama de terapia intermedia es de $\$ 790.00$ en salubridad o \$7,100.00 en un hospital privado tipo A1 con las mismas consideraciones en cuanto a gastos por hospitalización. Así, contar con un medicamento que provea un buen control del dolor y además ayude a evitar la intubación del paciente para toracoscopía disminuirá importantemente los gastos del paciente en hospitales privados y los gastos subsidiados por los impuestos en hospitales públicos.

\section{CONCLUSIONES}

1. El utilizar una analgesia multimodal durante la cirugía de toracoscopía disminuye un $200 \%$ la media para el dolor moderado-severo postquirúrgico inmediato.

2. Dosis de buprenorfina para prevención de dolor postquirúrgico entre 1 a $3 \mu \mathrm{g} / \mathrm{kg}$ no produce náusea, vómitos, RASH y/o depresión respiratoria.

3. No existen diferencias estadísticamente significativas entre el nivel de analgesia y/o sedación durante los primeros 120 minutos de ingreso a la UCPA entre las dosis de 1 a $3 \mu \mathrm{g} / \mathrm{kg}$.

4. El nivel de mantenimiento de analgesia fue más evidente con dosis de 2 y $3 \mu \mathrm{g} / \mathrm{kg}$ (30 minutos) que con dosis de 1 $\mu \mathrm{g} / \mathrm{kg}$ requiriendo mayor rescate analgésico.

5. La sedación con dosis de $1 \mu \mathrm{g} / \mathrm{kg}$ fue menor y se mantuvo por más tiempo, con dosis de $3 \mu \mathrm{g} / \mathrm{kg}$ se encontró mayor nivel de sedación (sin llegar a sedación profunda) pero con una rápida recuperación.

6. Al ser una muestra pequeña de pacientes, se requiere un ensayo clínico con mayor número de participantes.

\section{REFERENCIAS}

1. Dahl JB, Kehlet H. Postoperative pain and its management. Elsevier Churchill Livingstone; 2006: 635-651.

2. Hinrichs-Rocker A, Schulz K, Jarvinen I, Lefering R, Simanski C, Neugebauer EA. Psychosocial predictors and correlates for chronic postsurgical pain (CPSP): a systematic review. Eur J Pain. 2009;13:719730.

3. Martínez VA, Rodríguez BN. Posoperative pain: specific procedure approach. Revista de Ciencias Biomédicas 2012: 360-372.

4. Martínez-Vázquez CJ, Torres LM. Prevalencia del dolor posoperatorio. Alteraciones fisiopatológicas y sus repercusiones. Rev Soc Esp Dolor. 2000;7:465-476.

5. Chistopher L, Srinivasa N. Treatment of acute posoperative pain. Lancet. 2011;377:2215-2225.
6. Brennan T. Pathophysiology of posoperative pain. Pain. 2011;152:33-40.

7. Ji R, Kohno T, Moore K, Woolf C. La sensibilización central y LTP: hacer del dolor y la memoria comparten mecanismos similares? Trends Neurosci. 2003;26:696-705.

8. Bayman EO, Parekh KR, Keech J, Selte A, Brennan TJ. A prospective study of chronic pain after thoracic surgery. Anesthesiology. 2017;126:938-951.

9. González de Mejia N. Analgesia multimodal posoperatoria. Rev Soc Esp Dolor. 2005;12:112-118.

10. Serrano-Atero, Caballero J, Cañas A, García-Saura, Serrano-Álvarez C y Prieto J. Valoración del dolor. Rev Soc Esp Dolor. 2002;9:94-108.

11. Ferreira M, Pais J, Jensen M. Validity of four pain intensity rating scales. Pain. 2011;152:2399-2404. 
12. Neguss SS, Mello NK, Lisenmayer DC, Jones RM, Portoghese PS. Kappa opioid antagonist effects of the novel antagonist 5 '-guanidionaltrindole (GNTI) in an assay of shedule-controlled behavior in Rhesus monkeys. Psychopharmacology (Berl). 2002;163:412-419.

13. Kobayashi T, Chiba K, Tani M, Shimada N, Ishizaki T, Kuroiwa Y. Human buprenorphine N-dealkylation, is catalyzed by cytochrome P450 3a4. Drug Metabo Dispos. 1998;26:818-821.

14. Temel JS, Greer JA, Muzikansky A, Gallagher ER, Admane S, Jackson VA, et al. Early palliative care for patients with metastatic non-small-cell lung cancer. N Engl J Med. 2010;363:733.

15. Covarrubias-Gómez A. La necesidad de contar sistemas para la educación en dolor agudo. Rev Mex Anest. 2016;39:173-174.

16. Fields HL, Heinricher MM, Mason P. Neurotransmitters in nociceptive modulatory circuits. Annu Rev Neurosci. 1991;14:219-245.

17. Pergolizzi JR, Taylor R, Plancarte R, Bashkansky D y Muniz E. ¿Es la buprenorfina una buena opción en el manejo de dolor postoperatorio? Rev Soc Esp Dolor. 2012;19:281-292.

18. Grendelmeier P, Tamm M, Jahn K, Pflimlin E, Stolz D. Propofol versus Midazolam in Medical Thoracoscopy: A Randomized, Noninferiority Trial. Resp. 2014;88:126-136.
19. Bautista S, Guzmán O, Bojórquez-Sepúlveda HA, Sandoval-Virgen FG, Toracoscopía con anestesia local como procedimiento diagnóstico y terapéutico. Rev Med Md. 2012;3:217-222.

20. Licker M, Diaper J, Tschopp JM. Propofol: is it really worse than midazolam in medical thoracoscopy? Respiration. 2015;89:436.

21. Lu Yi-F, Hui HM, Hsu HH, Chen JS. Non-intubated thoracoscopic segmentectomy for second primary lung cancer in a patient with previous contralateral lobectomy and emphysematous bullae. J Cardiothorac Vasc Anesth. . 2016;30:1639-1640.

22. Ming-Hui Hung, Hsao-Hsun Hsu. Non-intubated thoracoscopic surgery using internal intercostal nerve block, vagal block and targeted sedation. Eur J Cardiothorac Surg. 2014;46:620-625.

23. Heel RC, Brogden RN, Speight TM, Avery GS. Buprenorphine: a review of its pharmacological properties and therapeutic efficacy. Drugs. 1979;17:81-110.

24. Hand C, Sear J, Uppington J, Ball MJ, McQuay HJ, Moore RA. Buprenorphine disposition in patients with renal impairment: single and continuous dosing, with special reference to metabolites. Br J Anesth. 1990;64:276-282. 